EPJ Web of Conferences 41, 03011 (2013)

DOI: $10.1051 /$ epjconf/20134103011

(C) Owned by the authors, published by EDP Sciences, 2013

\title{
Photoinduced femtosecond relaxation of antiferromagnetic orders in the iron pnictides revealed by ultrafast laser ellipsometry
}

\author{
A. Patz, ${ }^{1}$ T. Li, ${ }^{1}$ S. Ran, ${ }^{1}$ S. Bud'ko, ${ }^{1}$ P. Canfield ${ }^{1}$ and J. Wang ${ }^{1}$ \\ ${ }^{1}$ Ames Laboratory and Department of Physics and Astronomy, Iowa State University
}

\begin{abstract}
We report ultrafast softening of the antiferromagnetic order, $\sim 150 \mathrm{fs}$ after the electron thermalization, which follows a two-step recovery pathway to reveal a distinct interplay of magnetism and the nematic order in iron pnictides.
\end{abstract}

\section{INTRODUCTION}

Ultrafast studies of collective magnetic phenomena can provide fundamental insights into nonequilibrium processes of correlated spins. The key to understand and control these phenomena is the capability to directly reveal the spin fluctuations associated with the dynamic processes with femtosecond resolution, beyond time-averaged mean field values obtained from those static measurements. Although ultrafast spin spectroscopy and measurements are progressing well in ferromagnetic materials, femtosecond spin dynamics and dynamic critical phenomena of antiferromagnetic (AFM) orders are rarely addressed. AFM orders are extremely relevant for many "unconventional" materials since they are the key normal state properties out of which many elusive collective phenomena emerge, e.g., high-temperature superconductivity and colossal magnetoresistance. The correlation mechanisms determining the former are likely to be responsible for the latter. Thus far, most prior work on the ultrafast relaxation of AFM orders have focused on conventional, band insulators, where hundreds of ps magnetization quenching dynamics is revealed.

One prime example of such materials is the recently discovered high- $\mathrm{T}_{c}$ iron-based superconductors (FeSCs) [1]. FeSCs' exhibit a layered FeAs crystal structure with a complex phase diagram including: superconductivity, antiferromagnetism, Ising-nematic phase, orbital order, tetragonal and orthorhombic crystal structures [2]. One of the most perplexing behaviors of FeSCs occurs in the undoped (parent) and weakly doped antiferromagnetic compounds, where a significant in-plane anisotropy (broken $\mathrm{C} 4$ rotational symmetry) emerges in the temperature region between the striped AFM transition and the structural tetragonal-to-orthorhombic transition $\left(\mathrm{T}_{\mathrm{N}}<\mathrm{T}<\mathrm{T}_{\mathrm{S}}\right)$. Exactly what correlation mechanism can substantially break the symmetry above $\mathrm{T}_{\mathrm{N}}$ remains controversial. Thus far, experimental evidence for the anisotropic states in FeSCs has mostly been from stationary measurements, and has shown an onset of the anisotropy to appear well above $\mathrm{T}_{\mathrm{S}}$, which is inconsistent with the theoretical predictions of the Ising nematic order parameter [3]. Particularly, there is also a competing view for the roles of the spin vs. orbital orders as the origin of anisotropy.

This is an Open Access article distributed under the terms of the Creative Commons Attribution License 2.0, which permits unrestricted use, distribution, and reproduction in any medium, provided the original work is properly cited. 


\section{EXPERIMENTAL METHODS}

Here we explore dynamics of the AFM and anisotropic states that allow decoupling various correlations in the time domain to differentiate their contributions to the origin of the anisotropy. We have performed pump-probe experiments utilizing two-color ultrafast laser ellipsometry, $\Delta \mathrm{A}$, techniques to probe genuine anisotropy dynamics in parent and weakly Co-doped compounds of the Fe-pnictide superconductor family $\mathrm{BaFe}_{2} \mathrm{As}_{2}$. Our measurements directly reveal femtosecond spin relaxation time of photoexcited AFM orders, which exhibit distinct dependence on the transition temperatures, $\mathrm{T}_{\mathrm{N}}$ and $\mathrm{T}_{\mathrm{S}}$.

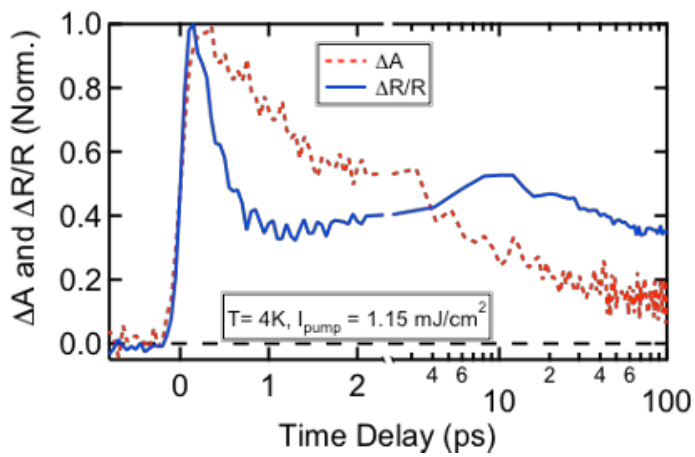

Fig. 1. Transient ellipticity $\Delta \mathrm{A}$ and $\Delta \mathrm{R} / \mathrm{R}$ are shown for the first $2 \mathrm{ps}$ and the extended time scales of 100 ps. The anisotropy and charge carriers show distinctly different relaxation dynamics in the time domain.

\section{RESULTS AND DISCUSSIONS}

A representative trace for the temporal profile of the ellipsometry and differential reflectivity, $\Delta \mathrm{R} / \mathrm{R}$, is shown in Figure 1 for the parent compound $\mathrm{BaFe}_{2} \mathrm{As}_{2}$ at $4 \mathrm{~K}$. There are noticeably different anisotropy and charge relaxation dynamics both in the first few ps and also at extended time scales of 100 ps. The anisotropy has the slower initial decay with a time-constant $\sim 2$ ps after photoexcitation compared to the charge relaxation which is on the order of $\sim 200 \mathrm{fs}$. The $\Delta \mathrm{R} / \mathrm{R}$ signal has some additional features not present in the $\Delta \mathrm{A}$ signals, such as a periodical oscillation and a strongly damped, ps component.

Figure 2(a) further shows the peak amplitude of the ellipticity signal as a function of temperature. It is clearly visible that the signal quickly diminishes as the temperature approaches $\mathrm{T}_{\mathrm{N}}$ $=136 \mathrm{~K}$, which indicates the anisotropy emerges at the onset of the antiferromagnetic order parameter. In accord, the anisotropy decay time-constant also shows a critical slowing down near $\mathrm{T}_{\mathrm{N}}$ and indicates three-dimensionality. For the parent compound, photoinduced anisotropy signals show similar relaxation profiles below $\mathrm{T}_{\mathrm{N}}$, as demonstrated by the normalized traces at two temperatures, $4 \mathrm{~K}$ and $130 \mathrm{~K}$.

We compared these results to that of an under-doped sample (4.7\% doping) where the spindensity-wave/structural transition temperatures are far apart. Most intriguingly, the ultrafast ellipticity signals for the $4.7 \%$ doping show distinct transitions at both $T_{\mathrm{N}} \sim 47 \mathrm{~K}$ and $\mathrm{T}_{\mathrm{S}} \sim 66 \mathrm{~K}$. The fs relaxation component only exists below $\mathrm{T}_{\mathrm{N}}$ and quickly diminishes above it, so we attribute this to the spin contributions. This provides a direct determination of the spin relaxation time of photomelted AFM orders. In addition, the distinct fs component clearly shows the AFM orders strongly contribute to the anisotropic nematic phase, which brings the system to the verge of a magnetic transition in the undoped compound, or occurs before a magnetic transition to induce a magnetostructural phase transition in the underdoped case. 

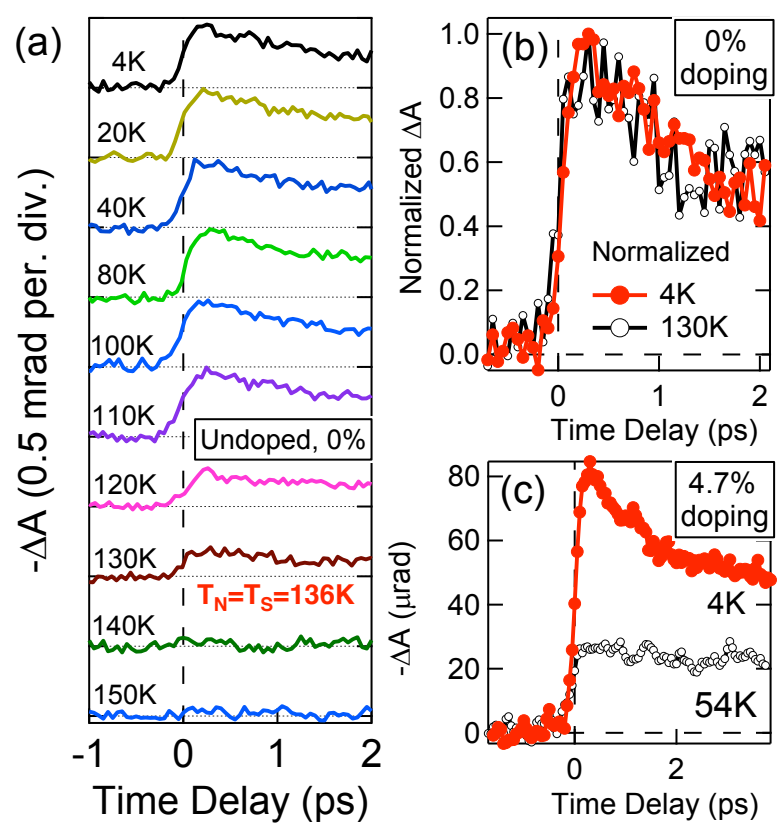

Fig. 2.(a) Temperature dependence of the transient ellipticity $\Delta \mathrm{A}$ for the parent compound BaFe2As2 $(0 \%)$ quickly diminishes with increasing the temperature and completely disappears at $\mathrm{T}_{\mathrm{S}} / \mathrm{T}_{\mathrm{N}}$. (b) Normalized $\Delta \mathrm{A}$ for the paraent compound at two temperatures, $4 \mathrm{~K}$ and $130 \mathrm{~K}$. (c) Transient ellipticity $\Delta \mathrm{A}$ for an under-doped sample $(4.7 \%$ doping) at two temperatures, $40 \mathrm{~K}$ and $54 \mathrm{~K}$. The AFM transition temperature is $\sim 47 \mathrm{~K}$.

\section{CONCLUSIONS}

In summary, we report fs spin relaxation in photoexcited undoped and weakly Cobalt doped $\mathrm{BaFe}_{2} \mathrm{As}_{2}$, which represents the first evidence to identify the dynamic interplay of antiferromagnetic correlation and the nematic phase in iron pnictides.

Acknowledgements. This work was also supported by the National Science Foundation Contract No. DMR-1055352. Material synthesis at the Ames Laboratory was supported by the U.S. Department of Energy-Basic Energy Sciences under Contract No. DE-AC02-7CH11358.

\section{References}

1. Kamihara, Y., Watanabe, T., Hirano, M., Hosono H., "Iron-based layered superconductor $\mathrm{La}\left[\mathrm{O}_{1-\mathrm{x}} \mathrm{F}_{\mathrm{x}}\right] \mathrm{Fe} A s(\mathrm{x}=0.05-0.12)$ with $\mathrm{T}_{\mathrm{c}}=26 \mathrm{~K}$,” J. Am. Chem. Soc. 130, 3296-3297 (2008).

2. J. Paglione and R.L. Greene, "High-temperature superconductivity in iron-based materials," Nature Phys. 6, 645 (2010).

3. R. M. Fernandes, A. V. Chubukov, J. Knolle, I. Eremin, and J. Schmalian, "Preemptive nematic order, pseudogap, and orbital order in the iron pnictides," Phys. Rev. B. 85, 024534 (2012). 